\title{
Adaptive Multi-Camera System for Real Time Object Detection
}

\author{
Massimo Camplani and Luis Salgado \\ Grupo de Tratamiento de Imágenes, Universidad Politécnica de Madrid, Spain
}

\begin{abstract}
In this paper we present an adaptive multi-camera system for real time object detection able to efficiently adjust the computational requirements of video processing blocks to the available processing power and the activity of the scene. The system is based on a two level adaptation strategy that works at local and at global level. Object detection is based on a Gaussian mixtures model background subtraction algorithm. Results show that the system can efficiently adapt the algorithm parameters without a significant loss in the detection accuracy.
\end{abstract}

\section{INTRODUCTION}

Object detection algorithms are fundamental modules in all video based tracking systems. The analysis of temporal and spatial information between different frames allows the detection of moving objects in a video sequence. Background subtraction is a technique that estimates a background model of the scene and, then, any deviation from the model is considered as a moving object. Different background modeling techniques have been presented in the literature [1].

Stauffer and Grimson [2] presented a popular algorithm that aims at estimating a background model of the scene where each pixel is independently modeled as a mixture of Gaussian distributions. The algorithm is composed by two fundamental steps: the first step is the estimation of whether or not a pixel belongs to the background model; during the second step the Gaussian parameters are updated. This "pixel-wise" approach is computational demanding since for each pixel all the parameters of the distributions have to be updated.

Several variants of this algorithm have been presented in the literature with the aim of reducing the computational time in real time applications [3]: improve the efficiency in the estimation of the parameters [4]; implement variable block size approaches, sub sampling techniques and hierarchical strategies [3]; or use a variable number of Gaussians per pixel as a function of the amount of foreground pixels [5]. Nevertheless, these approaches are strictly related to a specific application, showing a low degree of adaptability since they are based on the modification of only one characteristic of the algorithm. Their direct applicability to multi-camera systems is very limited, and, therefore, more efficient and adaptive strategies are required.

In this work we present an adaptive and efficient multicamera system for real time object detection able to adapt the computational requirements to the scene activity and the available processing resources.

This work has been supported by the Ministerio de Ciencia e Innovación of the Spanish Government under project TEC200767764 (SmartVision).

\section{SYSTEM OVERVIEW}

The proposed system (see Fig.1) is composed by a single processing unit (PU) connected to several cameras. The PU completes three different tasks. For each camera an acquisition task and one processing task are present. The acquired images are stored in a buffer. The controller unit is a centralized module that collects data on the overall system performance and manages the parameters adaptation phase.

The background subtraction algorithm is adaptively modified changing three different parameters: the number of Gaussian distributions per pixel; the frequency with which the parameters are updated and the image size: the algorithm can work at block-size level rather than at a pixel level.

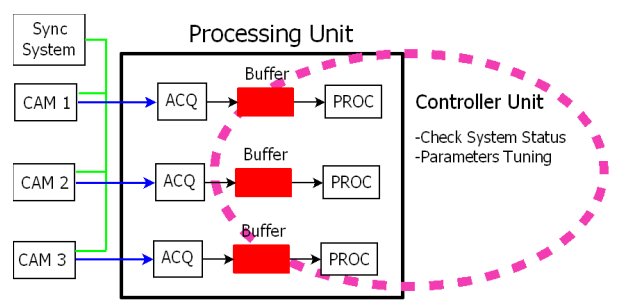

Fig. 1. Scheme of the proposed adaptive system.

\section{MULTI-LEVEL ADAPTATION STRATEGY}

The proposed parameters adaptation policy is a two level strategy. The first one works at local (camera) level: it adapts the background model parameters independently for each camera. If the changes done in the first level are not sufficient, the second (global) level balances the processing load among all the tasks. The buffer occupancy level is continuously monitored in order to have a measurement of the processing needs of each task: a growing level of buffer occupancy corresponds to a slow processing task.

Let us consider the $i_{t h}$ image buffer: we define $B_{\max }^{i}$ as the buffer size; $B_{u f l}^{i}$ and $B_{\text {ovfl }}^{i}$ as the minimum and maximum allowed buffer occupancy. We define $p T_{\max }^{i}$ as the processing time of the $i_{t h}$ processing module without any parameter adaptation (optimal algorithm) and $p T_{\min }^{i}$ as the minimum processing time of the $i_{\text {th }}$ processing module that does not compromise the detection accuracy. The target of the proposed strategy is to adapt the algorithm performance in order to prevent the buffer occupancy from exceeding $B_{u f f}^{i}$ and $B_{o v f l}^{i}$.

\section{A. Local Level Adaptation (LLA)}

There are two different strategies for parameters adaptation that depend on the scene (foreground) activity. If the scene 
shows mainly a static background, the current frame is said to be characterized by a Low Pixel Activity (LPA). When LPA is found, algorithm parameters are tuned to keep buffer occupancy close to $B_{u f l}^{i}$. First of all, the number of Gaussians used and the parameters update rate are decreased: in fact, a static background can be modeled with a smaller number of Gaussians without decreasing the detection accuracy and a fast update rate is not necessary. Finally, if buffer occupancy does not decrease to the desired level, spatial sub-sampling is applied. When moving objects have been detected, the current frame is said to be characterized by a High Pixel Activity (HPA). In this case a highly accurate algorithm is required: therefore, spatial sub-sampling is disabled, parameters update rate is increased and, if required, the number of Gaussian distributions is restored. These actions are done even if buffer occupancy increases; only when $B_{\text {ovf }}^{i}$ is reached, parameters are again modified to speed up the algorithm.

\section{B. Global Level Adaptation (GLA)}

Let us suppose that the $i_{\text {th }}$ processing task is working with a processing time very close to $p T_{\min }^{i}$ and buffer occupancy near $B_{\text {ovf }}^{i}$. Algorithm parameters cannot be further modified since this would dramatically decrease the background model quality and, therefore, the detection accuracy: then a global adaptive strategy is required. The processing task that is using more processing resources is forced to reduce its computation cost (i.e. enabling subsampling mode). Now, the $i_{t h}$ processing task can use these new available processing resources.

\section{EXPERIMENTAL RESULTS}

The system has been intensively tested in an indoor complex environment with three cameras connected to the PU. Each camera provides a stream of color images of $1032 \times 778$ pixels at $20 \mathrm{fps}$. The used detection algorithm is a variation of [2] presented by Power and Schoones in [4], and applied to the $\mathrm{YCbCr}$ space. Fig. 2 shows the results of the two level adaptation strategy: (a) shows an example of LLA where buffer occupancy (green line) and pixels activity (blue line) are reported. As it can be noticed, buffer occupancy is bounded between $B_{o v f l}^{i}$ and $B_{u f l}^{i}$ (dashed lines) and, while LPA is found, it maintains a value very close to $B_{u f l}^{i}$. When HPA is identified (frame 115), background model accuracy is ensured assigning more computational resources to the detection process (thus increasing buffer occupancy). As expected, the LLA strategy guarantees an efficient utilization of computational resources depending on the scene activity. Fig. 2(b) shows an example of GLA: when the buffer occupancy of camera 0 (black line) exceeds $B_{\text {ovf }}^{i}$, and the LLA cannot further reduce the processing time without compromising the detection accuracy (it is already working at $p T_{\min }^{i}$ ), the GLA reduces the computational requirements of the camera 1 processing module (blue line). The new available processing resources are given to camera 0 processing module, thus reducing its buffer occupancy. Fig. 3 shows, for an acquired original frame in (a), the detection result using the original algorithm (full processing requirements) in (b), and that when using the adaptive algorithm: moving object is clearly detected with only a moderate loss in its segmentation quality.
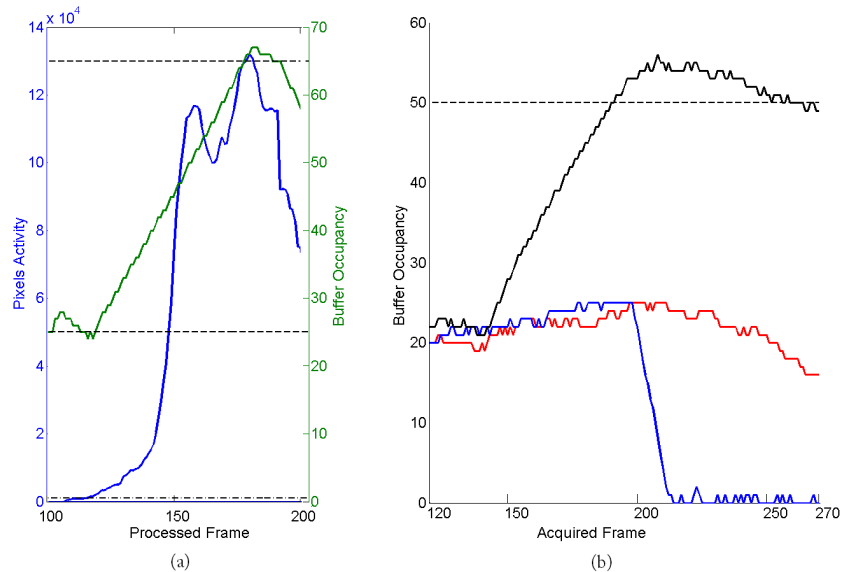

(b)

Fig. 2. Two level adaptation strategy example: (a) Buffer occupancy (green line) and scene activity (blue line); (b) buffer occupancy of the three buffers of the system.

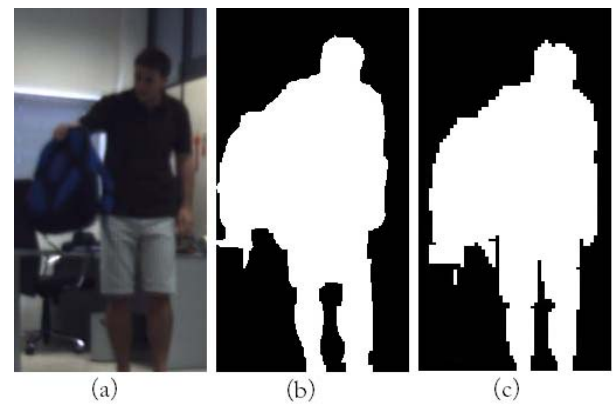

Fig. 3. Frame detail (a), object detected with the original algorithm (b), object detected with the adaptive system (c).

\section{CONCLUSIONS AND FUTURE WORKS}

In this work we present an adaptive multi-camera system for object detection based on a two level adaptation strategy that allows to dynamically tuning the processing requirements as a function of the scene activity and the available processing resources without affecting the algorithm accuracy. Next steps will address its integration with a real time 3D tracking system.

\section{REFERENCES}

[1] M. Piccardi. Background subtraction techniques: a review. In IEEE, International Conference on Systems, Man and Cybernetics, vol. 4, pp 3099-3104. October 2004.

[2] C. Stauffer, and W. E. L. Grimson, "Adaptive background mixture models for real-time tracking," IEEE Computer Society Conference on Vision and Pattern Recognition, vol.2, pp. 2246-2252, August 1999.

[3] T. Bouwmans, F. El Baf, B. Vachon, "Background Modeling using Mixture of Gaussian for Foreground Detection," Recent Patent on Computer Science, vol.1, 2008.

[4] P. Power, J. Schoones "Understanding background mixture models for foreground segmentation", Imaging and Vision Computing NewZeland 2002, November 2002.

[5] A. Shimada, D. Arita, R. Taniguchi, "Dynamic control of adaptive mixture-of-gaussian background model”. AVSS 2006. November 2006. 